\title{
NEW INSIGHTS INTO THE HYPOTHALAMIC-PITUITARY-THYROID AXIS
}

\author{
L.H. Duntas ${ }^{1, *}$ \\ University of Athens, Evgenideion Hospital, Unit of Endocrinology, Diabetes and Metabolism, Thyroid Section, \\ Athens, Greece
}

\begin{abstract}
The discovery of thyrotropin releasing hormone (TRH) in 1969 was the definitive step in decoding the hypothalamic-pituitary thyroid (HPT) axis, thereby opening up the era of neuroendocrinology, while it also revolutionized the diagnostic and therapeutic approach to patients with thyroid diseases. TRH, produced in the hypothalamus, is the central regulator of the HPT. It functions via neurons originating in the paraventricular nucleus (PVN), which integrates multiple neuronal and humoral signals and resets the HPT axis according to variations of external and internal environmental conditions. The TRH activates TSH in the pituitary that stimulates the secretion of thyroxine from thyroid which, in turn, exerts a negative feedback on TSH and TRH secretion. However, various factors are involved in the regulation of the HPT axis.

Leptin has both indirect and direct effects on TRH regulation, the former by regulating agouti-related peptide (AGRP) in the arcuate nucleus (ARN) that antagonizes the $\alpha$-MSH stimulatory activity on pro-TRH gene expression in the PVN, and the latter by stimulating hypothalamic TRH expression, TRH transcription via stimulation of proconvertase 1 and 2 expression, which lead to enhanced processing of pro-TRH into TRH. The interplay of TRH with leptin and the recently reported influence of ghrelin on the HPT axis can alter the setpoint of the axis.

The polyphenol resveratrol, as recently observed, exerts an anxiolytic and antidepressant activity in subclinical hypothyroid $(\mathrm{SCH})$ rats. Resveratrol, by decreasing both TSH and TRH mRNA expression, regulates the HPT axis, while in parallel it regulates the $\mathrm{Wnt} / \beta$-catenin pathway in the hippocampus. These findings open up possibilities for the therapeutic use of resveratrol as coadjuvant, especially in overt and $\mathrm{SCH}$ states marked by anxiety and depression. The clinician should be aware of clinical changes that can invalidate the normal regulation of the HPT axis, the most commonly observed being medications and comorbidities.
\end{abstract}

Key words: Hypothalamic-Pituitary Thyroid axis, TRH, TSH, tanycytes.

\section{INTRODUCTION}

"The isolation and characterization of TRF was the major event in the establishment of modern neuroendocrinology, the inflection point that separated confusion and a great deal of doubt from real knowledge."

Roger Guillemin(1)

The discovery of thyrotropin releasing hormone (TRH) in 1969 not only vindicated many years of hard work within the paradigm of a hypothalamic neurohormonal control of adenohypophysis secretion, but it was the most heuristic event in establishing the science of neuroendocrinology (1-3). It was the unequivocal demonstration of Geoffrey Harris' theory (1955) that the capillary network from the median eminence does not flow into the general circulation, but is conveyed by tiny blood vessels that discharge into the pituitary stalk, subsequently terminating in a second capillary network in the anterior pituitary gland (4). Harris postulated that this portal system provides a link via which shorter range hypothalamic hormones are transported to the anterior pituitary where they stimulate the secretion of anterior pituitary hormones into the systemic circulation.

$\mathrm{TRH}$, by activating thyrotropin (TSH) secretion from the pituitary thyrotrophs, stimulates the secretion of thyroid hormones which subsequently exert an inhibitory effect on TRH and TSH secretion, thereby setting the stage for negative feedback regulation, It is thus evident that TRH plays a leading role in the regulation of the HPT axis. In line with this evidence, deletion of the TRH gene in mice results in central hypothyroidism, while in humans central hypothyroidism is caused by TRH receptor mutations $(5,6)$.

*Correspondence to: Leonidas H Duntas, University of Athens, Evgenidion Hospital, Unit of Endocrinology, Diabetes and Metabolism. Thyroid Section, Medical School, Papadiamantopoulou 20, Athens 11528, Greece, E-mail: ledunt@otenet.gr 
The hypothalamic-pituitary thyroid (HPT) axis was the first neuroendocrine axis to be identified, a major discovery which introduced the era of neuroendocrinology and transformed the diagnostic and therapeutic approach in patients with thyroid disease.

The aim of this review is to briefly examine the vital role and clinical significance of the HPT axis. Several new findings are described that highlight the remarkable part played by TRH in energy metabolism, thermogenesis and feeding behavior, while its well established regulatory function in thyroid homeostasis is also discussed.

\section{REGULATION AND METABOLISM}

TRH is synthesized in the parvocellular region of the paraventricular nucleus (PVN) and, via hypophysiotropic neurons, which are located exclusively in the periventricular and medial parvocellular subdivisions of the PVN, is conveyed to and released into the median eminence (ME) in the third ventricle (7). From the ME, TRH is transported through the portal system into the anterior pituitary where it stimulates the secretion of pituitary TSH (Fig. 1). TSH is not only regulated by feedback from thyroid hormones in the pituitary (long feedback) and the hypothalamus, but also by feedback of the hypophyseal hormone at the hypothalamic (short feedback) and the pituitary (ultra-short feedback) level $(8,9)$. However, the multiple feedback loops on several levels of organization, with different time scales, optimal conditions of operation may reduce the use of TSH measurement, although valuable in many situations, to a supporting role (10). This reopens the debate on the measurement of free thyroid hormones and encourages more analytical studies to define the biomarkers.

Recently, the regulation of the pyroglutamyl peptidase II (PPII), which is a metallopeptidase that degrades TRH in the extracellular space, was analyzed in the mediobasal hypothalamus of adult rats (11). PPII mRNA was localized in tanycytes, which are cells lining the floor and infralateral walls of the third ventricle and are extended in a linear fashion to the external zone of the median eminence tightly apposed to pro-TRH-containing axon terminals (11). When animals were rendered thyrotoxic, a dramatic increase of up to $80 \%$ in PPII activity and subsequently TRH degradation was observed. In contrast, PPII inhibition by IP injection of a specific PPII inhibitor in the mediobasal hypothalamus enhanced TRH secretion and increased cold-, stress- and TRH-induced TSH levels in plasma (11). Therefore, it is likely that an increase in circulating thyroid hormone up-regulates PPII activity in tanycytes and enhances degradation of extracellular TRH in the ME, thereby considerably contributing to the feedback regulation of TSH by thyroid hormones.

$\mathrm{T} 3$ in the brain originates from local T4 to T3 conversion by an enzymatic outer ring deiodination of $\mathrm{T} 4$, primarily catalyzed in the central nervous system by type 2 iodothyronine deiodinase (DIO2) (12). Within the hypothalamus, DIO2 activity is highly expressed

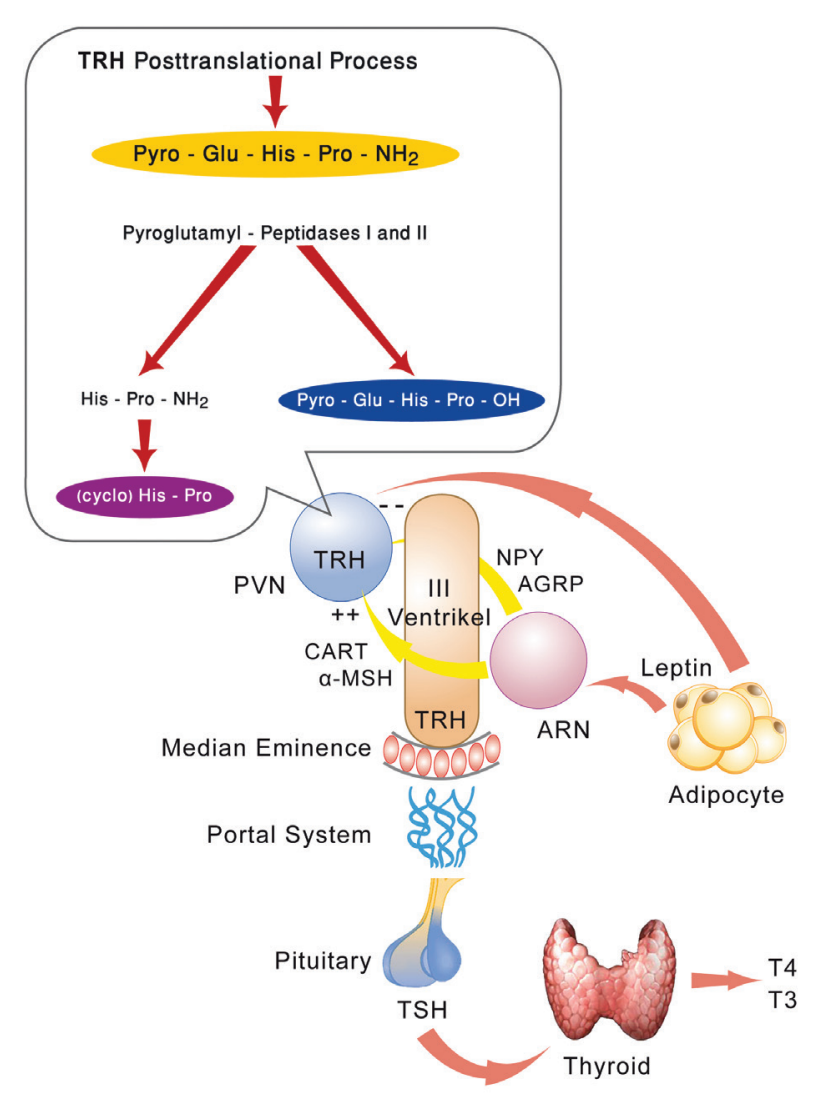

Figure 1. In the posttranslational process, TRH is degraded by the pyroglutamyl-peptidases I and II to its main metabolite His-Pro-NH2, which can be spontaneously metabolized to cyclo (His-Pro), and by the action of prolyl endopeptidase (TRH desamidase) to desamidated pyro-Glu-His-Pro-OH. TRH is released by hypophysiotopic neurons in the median eminence from where it is transported by the portal plexus in the anterior pituitary where it stimulates TSH secretion that stimulates the secretion of T4 and T3 from the thyroid cells. Potent orexigenic peptides, neuropeptide $Y$ (NPY) and agouti-related peptide (AGRP), that are projected from the arcuate nucleus (ARN) to the paraventricular nucleus (PVN) and innervate TRH neurons, are inhibited by leptin. In contrast, the neuronal group that synthesizes the anorectic peptides, $\alpha$-melanocyte-stimulating hormone ( $\alpha-\mathrm{MSH})$ and cocaine- and amphetamine regulated transcript (CART), is stimulated by leptin. Thus, leptin, by inhibiting NPY and AGRP, which antagonizes the $\alpha$-MSH stimulatory activity on pro-TRH gene expression in the $\mathrm{PVN}$, activates pro-TRH expression. 
in the region of the mediobasal hypothalamus $(\mathrm{MBH})$, specifically in the ME, ARN and ventromedial nucleus. The tripeptide TRH is derived by proteolytic cleavage of a larger precursor via the prohormone convertases 1 and 2 (PC1 and PC2) (13, 14). Prohormone TRH (pro-TRH) processing occurs in two phases: a) during axonal transport after removal of the signal peptide in the endoplasmic reticulum and b) as intermediate peptides move down the axon toward the nerve terminal $(15,16)$. Thus the proteolytic process of the pro-TRH continuum starts in the endoplasmatic reticulum and ends as a post-Golgi event. It should be stated that the posttranslational processing of various neuropeptides is critical in the regulation of energy balance and in the pathogenesis of obesity. Leptin and other physiological changes affect the biosynthesis and processing of many hormones as well as the regulation of the prohormone convertases responsible for the maturation

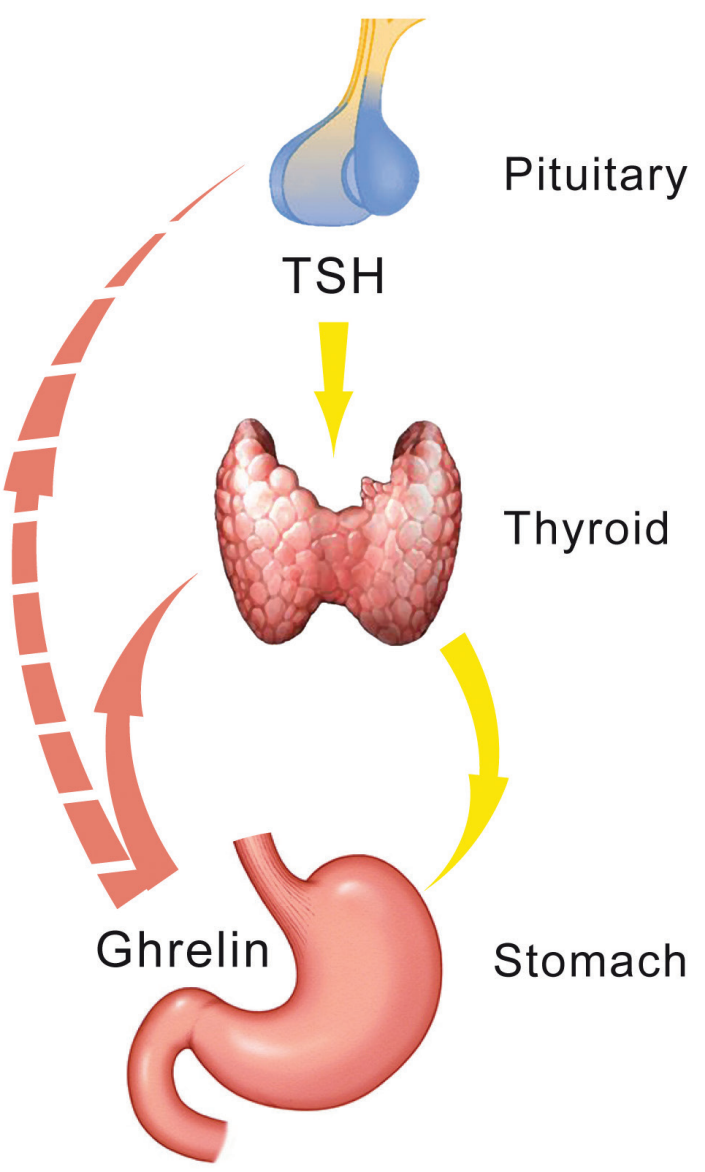

Figure 2. Ghrelin is secreted in the stomach and in turn stimulates the secretion of T4. It is not clear whether the reported inhibition of TSH following ghrelin administration is the result of a direct effect of ghrelin at the pituitary level or comes about via the inhibition by the thyroid hormones (negative feedback). Ghrelin can activate the orexigenic peptides AGRP and NPY in the ARN, thus exhibiting an antagonistic action to leptin. of these hormones. It is conceivable that regulation of energy balance by leptin requires the regulation of several pro-neuropeptides such as pro-TRH and proopiomelanocortin (POMC) (Fig. 2). In ARN there are two distinct populations of neurons that are directly regulated by leptin and which are strongly involved in the regulation of feeding behavior and project to TRH neurons in the PVN (17). The first is a neuronal group that synthesizes two potent orexigenic peptides, neuropeptide Y (NPY) and agouti-related peptide (AGRP), that are inhibited by leptin. The second is a neuronal group that synthesizes the anorectic peptides, $\alpha-\mathrm{MSH}$ and cocaine- and amphetamine-regulated transcript (CART) that are stimulated by leptin (17). Both NPY and AGRP-containing fibers densely innervate all TRH neurons in the PVN (18). Leptin, by regulating NPY and AGRP in the ARN, which antagonizes the $\alpha$-MSH stimulatory activity on proTRH gene expression in the PVN, indirectly regulates pro-TRH expression. Concomitantly, leptin stimulates PC1 and PC2 mRNA and protein expression, while starvation results in low serum leptin levels and decreases PC1 and PC2 gene and protein expression in the paraventricular nucleus (PVN) (18). Hence, leptin exhibits a direct, though modest, stimulatory effect on hypothalamic TRH generation.

In pathologic states, such as in hypothyroidism, the expression of orexigenic neuropeptide Y (NPY) and anorexigenic proopiomelanocortin (POMC) are modulated independently of the nuclei-specific changes in hypothalamic leptin signaling (19). As a result, hypothyroidism results in hypophagia, decreases the protein content of NPY and increases the content of POMC in the ARN, while it induces central resistance to the acute anorexigenic effect of leptin. In this line of evidence, while euthyroid rats displayed reduced food intake after leptin i.c.v. injection, hypothyroid rats showed no response (19).

The PVN receives and integrates multiple neuronal signals and adjusts the HPT axis to external and internal environmental changes, such as cold, starvation and illness. The input of adrenergic neurons from the brainstem into the hypophysiotropic TRH neurons with which they form stimulatory synapses, mediate the stimulatory effects of cold exposure on the hypophysiotropic TRH neurons (20). The rapid peak of TRH synthesis induced during cold exposure is associated with an increase in the hypothalamic concentration of catecholamines. Since an increase in thyroid hormone normally inhibits TRH gene transcription at the level of the PVN via negative 
feedback effects, catecholamines may increase the setpoint for inhibition of TRH gene expression by T3, thereby permitting high circulating levels of thyroid hormone to contribute to increased thermogenesis (20).

Thus, nutritional status regulates TRH neurons activity, this occurring via neurons located in the ARN that are sensitive to metabolic signals, for example, circulating leptin levels. In addition, energy-demanding situations like exercise and cold, activate TRH expression and the HPT axis, while, by contrast, states of energy balance, including fasting, inflammation and chronic stress, inhibit their activity.

\section{CLINICAL ASPECTS}

The HPT axis, which is critical to maintaining the euthyroid state and metabolic homeostasis, can be investigated reliably via TSH measurement. The TRH-test has been used for a quarter of century as the "gold standard" for studying the TSH-response after stimulation with TRH and, subsequently, thyroid function. However, in the past 20 years the establishment of the third and fourth generation assays for the measurement of TSH has superseded the use of the TRH-test in the diagnosis of thyroid diseases (21, 22).

Currently, the test could occasionally be useful in, for example, patients with central hypothyroidism to distinguish between pituitary and hypothalamic etiology. Basal TSH in these patients is surprisingly normal or slightly decreased in conjunction with low FT4 levels (23). In some cases it can be helpful combined with imaging and biochemical and clinical findings in the diagnosis of central hypothyroidism. In euthyroid sick syndrome (ESS), which is characterized by low T3, normal or low T4/FT4 and paradoxically normal basal TSH levels, the TSH response to TRH administration is blunted, which later renders the test practically useless in this situation. Severe ESS and acute administration of dopamine or glucocorticoids may suppress TSH even below $0.1 \mathrm{mU} / \mathrm{L}$. The TRH-test might have a certain validity in acromegaly in which a number of patients exhibit an inappropriate increase in $\mathrm{GH}$ secretion not related to the basal GH levels. Also in other pituitary diseases, such as Cushing's disease and Nelson syndrome, TRH testing may be used, despite its low specificity, as an alternative when other tests are not available. In patients with prolactinomas, the prolactin response to TRH exhibits a wide variation, which renders the test less useful. In patients with TSH-secreting tumors the TSH response is absent with an increase in the $\alpha$-subunit of the TSH.

In recent animal studies, it was reported that the polyphenol compound resveratrol may decrease TSH secretion and the elevated TRH mRNA in hypothyroid rats with anxiety and depression-like behavior (24), while by improving the $\mathrm{Wnt} / \beta$-catenin pathway in the hippocampus, it ameliorates mood and anxiety (24).

In another study the administration of ghrelin resulted in an increase of T4 and a blunting of TSH, though it is not clear whether this is due to a direct effect of ghrelin on TSH secretion or to the negative feedback of thyroid hormones at the pituitary level (25). The findings of these studies are paradigms demonstrating to what extent the setpoint may be changed by various compounds and drugs.

The latest discoveries showing the cardinal importance of the HPT axis and especially of TRH as the central mediator in energy homeostasis, besides its capital role in the regulation of thyroid function, have focused even greater emphasis on the importance of the HPT for human health. The clinician must be aware that several conditions, including stress, fasting, cold exposure and various compounds and drugs, may variably change the setpoint for inhibition or activation by thyroid hormones.

\section{Conflict of interest}

The author declares that he has no conflict of interest concerning this article.

\section{References}

1. Guillemin R. Characterization of the thyrotropin releasing factor, now known as thyrotropin releasing hormone. Reminiscences and reflections thirty years later. In: Hypothalamic Releasing Hormones. Challenges and Perspectives at the 2000 year mark. Eds. Duntas LH, Guillemin R, Beta Medical Art, Athens, 2000; 7-16.

2. Burgus R, Dunn RF, Desiderio D, Guillemin R. Stucture moleculaire du facteur hypothalamique hypophysiotrope TRF d'origine ovine: evidence par spectometrie de masse de la seequence PGA-His-Pro-NH2. CR Acad Sci (Paris) 1969 ; 269: 1870-1873.

3. Schally AV, Redding TW, Bowers CY, Barett JF. Isolation and properties of porcine thyrotropin-releasing hormone. J Biol Chem 1969; 244: 4077-4081.

4. Geoffrey Raisman. 60 years of neuroendocrinology: Memoir: Geoffrey Harris and my brush with his unit. J Endocrinology 2015; 226 (2): T1-T11.

5. Yamada M, Saga Y, Shibusawa N, Hirato J, Murakami M, Iwasaki T, Hashimoto K, Satoh T, Wakabayashi K, Taketo MM, Mori M. Tertiary hypothyroidism and hyperglycemia in mice with targeted disruption of the thyrotropin-releasing hormone gene. Proc Natl Acad Sci U S A. 1997; 94(20):10862-10867.

6. Beck-Peccoz P, Persani L, Calebiro D, Bonomi M, Mannavola $\mathrm{D}$, Campi I. Syndromes of hormone resistance in the hypothalamicpituitary-thyroid axis. Best Pract Res Clin Endocrinol Metab. 2006; 20(4):529-546. 
7. Swanson LW, Kuypers HG. The paraventricular nucleus of the hypothalamus: cytoarchitectonic subdivisions and organization of projections to the pituitary, dorsal vagal complex, and spinal cord as demonstrated by retrograde fluorescence double-labeling methods. J Comp Neurol 1980; 194:555-570.

8. Prummel MF, Brokken LJ, Wiersinga WM. Ultra shortloop feedback control of thyrotropin secretion. Thyroid. 2004; 14(10):825-829.

9. Fekete C, Lechan RM. Central regulation of hypothalamicpituitary-thyroid axis under physiological and pathophysiological conditions. Endocr Rev. 2014; 35(2):159-94.

10. Hoermann R, Midgley JE, Larisch R, Dietrich JW. Homeostatic control of the thyroid-pituitary axis: perspectives for diagnosis and treatment. Front Endocrinol (Lausanne). 2015; 20; 6:177.

11. Sánchez E, Vargas MA, Singru PS, Pascual I, Romero F, Fekete C, Charli JL, Lechan RM. Tanycyte pyroglutamyl peptidase II contributes to regulation of the hypothalamic-pituitary-thyroid axis through glial-axonal associations in the median eminence. Endocrinology. 2009; 150 (5):2283-2291.

12. Bianco AC, Salvatore D, Gereben B, Berry MJ, Larsen PR. Biochemistry, cellular and molecular biology, and physiological roles of the iodothyronine selenodeiodinases. Endocr Rev 2002;23:38-89.

13. Sanchez VC, Goldstein J, Stuart RC, Hovanesian V, Huo L, Munzberg H, Friedman TC, Bjorbaek C, Nillni EA. Regulation of hypothalamic prohormone convertases 1 and 2 and effects on processing of prothyrotropin releasing hormone. J Clin Invest. 2004; 114(3): 357-369.

14. Nillni EA, Luo LG, Jackson IM, McMillan P. Identification of the thyrotropin-releasing hormone precursor, its processing products, and its coexpression with convertase 1 in primary cultures of hypothalamic neurons: anatomic distribution of PC1 and PC2. Endocrinology 1996; 137(12):5651-5661.

15. NillniEA. Regulation of prohormone convertases in hypothalamic neurons: implications for prothyrotropin-releasing hormone and proopiomelanocortin. Endocrinology 2007;148(9):4191-4200.

16. Duntas LH, Emerson C. Thyrotropin-releasing hormone: shedding new light on the hypothalamic-pituitary thyroid axis. HotThyroidology 2009; 17: 1-16.
17. Lechan RM, Fekete C. The TRH neuron: a hypothalamic integrator of energy metabolism. Prog Brain Res. 2006; 153:209235.

18. Nillni EA, Vaslet C, Harris M, Hollenberg A, Bjørbak C, Flier JS. Leptin regulates prothyrotropin-releasing hormone biosynthesis. Evidence for direct and indirect pathways. J Biol Chem. 2000 Nov 17;275(46):36124-33.

19. Calvino C, Império GE, Wilieman M, Costa-E-Sousa RH, Souza LL, Trevenzoli IH, Pazos-Moura CC. Hypothyroidism induces hypophagia associated with alterations in protein expression of neuropeptide $\mathrm{Y}$ and proopiomelanocortin in the arcuate nucleus, independently of hypothalamic nuclei-specific changes in leptin signaling. Thyroid. 2016; 26(1):134-43.

20. Joseph-Bravo P, Jaimes-Hoy L, Uribe RM, Charli JL. 60 years of neuroendocrinology: TRH, the first hypophysiotropic releasing hormone isolated: control of the pituitary-thyroid axis. J Endocrinol. 2015; 226(2):T85-T100.

21. Anderson MS, Bowers CY, Kastin AJ, Schalch DS, Schally AV, Snyder PJ, Utiger ED, Wilber JF, Wise AJ. Synthetic thyrotropinreleasing hormone. A potent stimulator of thyrotropin-secretion in man. New Engl J Med 1971; 285: 1279-1283.

22. Faglia G. The clinical impact of thyrotropin- releasing hormone test. Thyroid 1998; 10: 903-908.

23. Beck-Peccoz P, Amir S, Menezes-Fereira MM, Faglia G, Weintraub BD. Decreased receptor binding in biologically inactive thyrotropin in central hypothyroidism: Effect of treatment with thyrotropin-releasing hormone. N Engl J Med 1985; 312: 10851090.

24. Jin-Fang Ge, Ya-Yun Xu, Gan Qin, Jiang-Qun Cheng and Fei$\mathrm{Hu}$ Chen. Resveratrol ameliorates the anxiety- and depression-like behavior of subclinical hypothyroidism rat: possible involvement of the HPT axis, and Wnt/ $\beta$-catenin pathway. Front. Endocrinol. 2016;7(44):1-11.

25. Kluge M, Riedl S, Uhr M, Schmidt D, Zhang X, Yassouridis A, Steiger A. Ghrelin affects the hypothalamus-pituitary-thyroid axis in humans by increasing free thyroxine and decreasing TSH in plasma. Eur J Endocrinol. 2010 Jun;162(6):1059-65. 